\title{
Therapeutic effects of photobiomodulation in the speech-language-hearing clinic: an integrative literature review
}

Viviane Souza Bicalho Bacelete ${ }^{1}$ https://orcid.org/0000-0002-4483-4125

Ana Cristina Côrtes Gama ${ }^{2}$ https://orcid.org/0000-0002-7814-5328

Universidade Federal de Minas Gerais UFMG, Faculdade de Medicina, Programa de Ciências Fonoaudiológicas, Belo Horizonte, Minas Gerais, Brasil.

Universidade Federal de Minas Gerais UFMG, Faculdade de Medicina, Departamento de Fonoaudiologia, Belo Horizonte, Minas Gerais, Brasil.

This study was carried out in the Department of Speech-Language-Hearing Sciences at the Faculdade de Medicina da Universidade Federal de Minas Gerais UFMG, Belo Horizonte, Minas Gerais, Brazil.

Research support source: Coordenação de Aperfeiçoamento de Pessoal de Nível Superior -Brasil (CAPES) - Finance Code 001. Conselho Nacional de Desenvolvimento Científico e TecnológicoBrasil (CNPq - no. 309108/2019-5).

Conflict of interests: Nonexistent

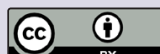

Received on: July 23, 2020

Accepted on: January 5, 2021

Corresponding address:

Viviane Souza Bicalho Bacelete Avenida Alfredo Balena, 190, sala 249 ,

Santa Efigênia

CEP: 30130-100 - Belo Horizonte,

Minas Gerais, Brasil

E-mail: vivisouzafono@yahoo.com.br

\section{ABSTRACT}

Purpose: to conduct a literature review on the therapeutic effects of photobiomodulation applicable to speech-language-hearing therapy.

Methods: the databases searched were the Cochrane Library, Virtual Health Library, Medical Literature Analysis and Retrieval System Online (MEDLINE) via PubMed, and Web of Science/ISI.The selected articles were original ones whose abstracts were available and that evaluated the therapeutic effect of photobiomodulation in situations related to speech-language-hearing practice.

Results: the sample comprised 23 articles, most of them being indexed in PubMed. The fields of health with the largest number of publications were Physical Therapy and Medicine. The sample size ranged from 1 to 99 people, aged 15 to 77 years, and the most applied wavelength was the infrared one. Most of the studies had positive photobiomodulation application results - although in a few publications the effects of this treatment modality were assessed in a combination with rehabilitation exercises.

Conclusion: photobiomodulation benefits different disorders treated by speech-language-hearing therapists, however, there is a broad methodological diversity, lacking specific protocols for the ideal dosimetry for each disorder.

Keywords: Audiology; Speech, Language and Hearing Sciences; Stomatognathic System; Low-Level Light Therapy; Voice 


\section{INTRODUCTION}

Low-level laser (LLL) therapy to modulate cell and tissue physiology can be applied from light sources such as a light-emitting diode (LED) and low-level light amplification by stimulated emission of radiation (laser) ${ }^{1}$.

Photobiomodulation (PBM) is the application of light to a biological system to induce a photochemical process - especially in the mitochondria, stimulating the production of energy as adenosine triphosphate $(\text { ATP })^{2}$. Hence, cell metabolism is increased, producing effects such as analgesia ${ }^{3}$, tissue regeneration, wound scarring $^{4}$, decreased muscle fatigue ${ }^{5}$, and so forth. Besides these, evidence from the application of neuromodulation to different regions of the nervous system points to an increase in brain perfusion, resulting in cognitive and behavioral improvement in neurological diseases such as dementia, traumatic conditions, and Parkinson's disease, besides possible cognitive improvements in healthy people ${ }^{6}$.

Speech-language-hearing therapists are increasingly interested in joining the group of professionals who use PBM, as it is a noninvasive and nontoxic therapeutic resource with promising results. Some studies have recently evaluated its results in speech-languagehearing rehabilitation in the fields ofAudiology and Oral-Motor Function and observed, respectively, reduced tinnitus ${ }^{7}$ and improved electrical activity in the orbicularis oris muscle ${ }^{8}$.

Despite the scarcity of studies in speech-languagehearing pathology, the application of PBM in disorders also treated by speech-language-hearing therapists has already been reported in the literature. In Audiology, there are studies on tinnitus ${ }^{9,10}$ and hearing loss $^{11}$. In Language, there is research on the use of neuromodulation in healthy people ${ }^{12}$ andischemic ${ }^{13}$, neurodegenerative ${ }^{13,14}$, and traumatic disease ${ }^{15}$ reporting improved naming capacity in aphasic patients, as well as improvements in other cognitive skills ${ }^{16}$. In Oral-Motor Function, which concentrates a large part of the research, temporomandibular disorder (TMD) ${ }^{17-22}$, nipple fissures ${ }^{23-25}$, and facial palsy (FP) ${ }^{26-30}$ treatment is highlighted. Lastly, in the field of Voice, a study has been conducted on vocal fatigue ${ }^{31}$.

Even though PBM has been used in the speechlanguage-hearing clinic, there is no robust evidence of its effects as a therapeutic technique, considering clinical aspects such as 1) most effective application (before, during, after exercises); 2) wavelength to be used; 3) application time; and 4) dosimetric parameters.
Therefore, speech-language-hearing interventions with PBM application need to be standardized, using structured clinical protocols for the rehabilitation of different clinical conditions.

Given the above, this study aimed to conduct a literature review on the PBM therapeutic effects applicable to speech-language-hearing therapy.

\section{METHODS}

\section{Research strategies}

This is an integrative literature review. The studies were selected based on the following steps: development of the research question, search in the literature, and critical analysis of the studies ${ }^{32}$.

The following research question guided the search on which the review was based: What are the therapeutic effects of PBM on disorders treated by speechlanguage-hearing therapists?

The articles were selected from the Cochrane Library, Virtual Health Library (VHL), Medical Literature Analysis and Retrieval System Online (MEDLINE) via PubMed, and Web of Science/ISI databases. The descriptors used were "Audiology", "Speech, Language and Hearing Sciences", "Stomatognathic System", "Low-Level Light Therapy", and "Voice", and their combinationsin English, Portuguese, and Spanish. The other speech-language-hearing specializations, as they are not descriptors, were used in the search as free terms.

\section{Selection criteria}

After finding the studies, they were screened by title and abstract. This stage was independently carried out by two speech-language-hearing evaluators. Considering the inclusion and exclusion criteria,23 articles were selected to be read in full.

Original articles whose full text was available, published between 2010 and 2020, assessing the therapeutic effects of PBM in situations applicable to speech-language-hearing therapy were selected.

Duplicated articles, found in more than one database, were excluded, as well as those evaluating only analgesia or other PBM effects, not considering functional aspects, studies in animals, and ongoing research, whose final results had not yet been published.

Hence, the sample comprised 23 articles, of which 19 were inMEDLINE/ PubMed and four, inVHL (Figure 1). 

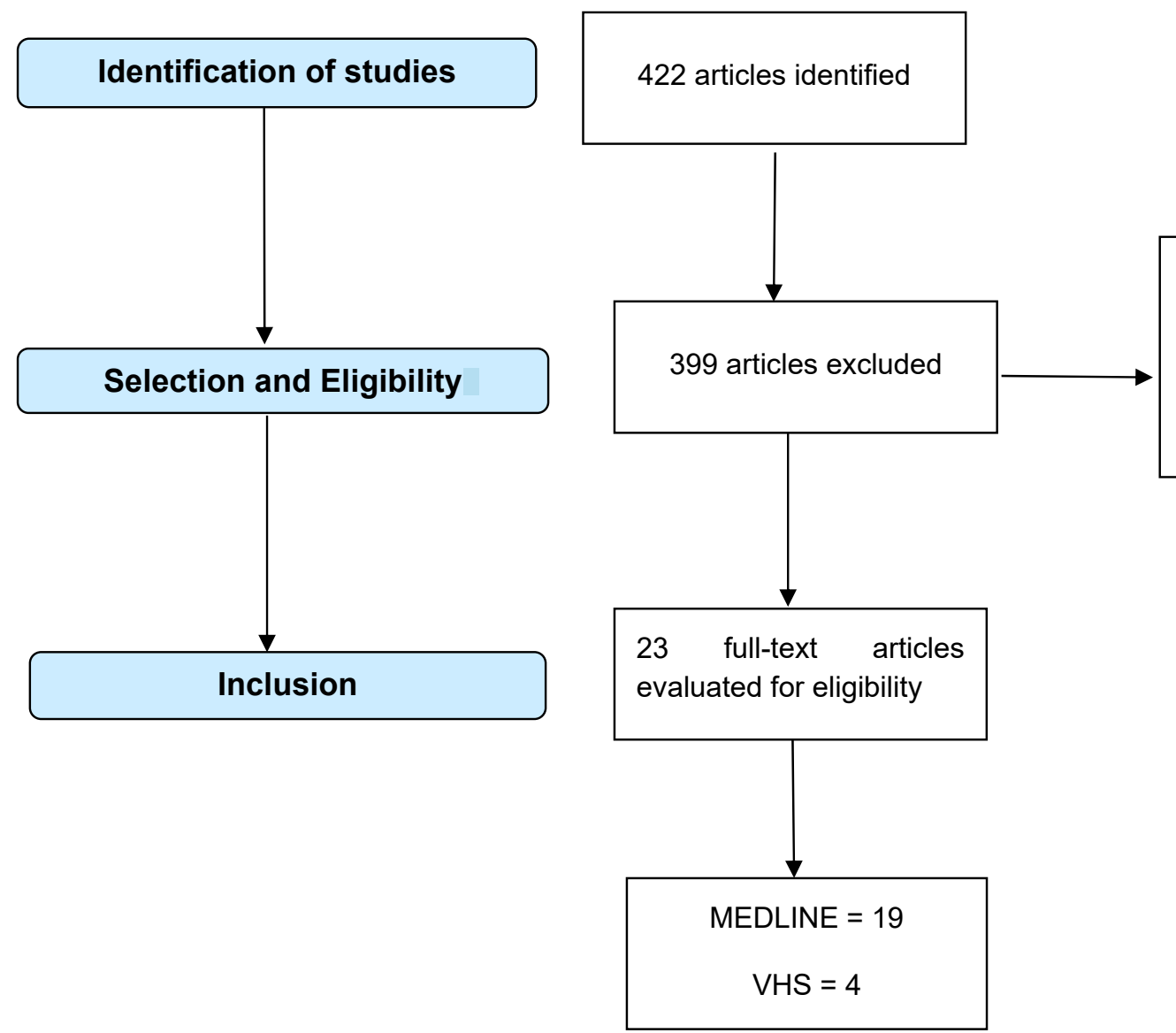

Duplicated articles

Ongoing studies

Research on animals or that evaluated only analgesia

Figure 1. Selection and analysis of the articles

The texts were fully read and analyzed considering year and place of publication, language, first author's professional education, study design, classification of the level of scientific evidence by the Oxford scale $^{33}$, objectives, sample, types of PBM, wavelength, number of sessions, areas of application, energy per point, time of application, and outcomes in each identified theme. The Oxford scale $^{33}$ classifies, particularly, the study designs; the evidence is classified in levels, from best to worst, as 1a, 1b, 1c, 2a, 2b, 2c, 3a, 3b, 4, and 5 .

\section{LITERATURE REVIEW}

Based on the methodology applied, 23 references were selected -10 national and 13 international articles. Regarding language, 21 articles had been published in English (91.3\%), one in Portuguese (4.35\%), and one in Spanish (4.35\%). Brazil led the number of articles, followed by the United States (Figure 2).

As for the year, a larger number of publications were from 2012 and 2019 (Figure 3). 


\section{4}

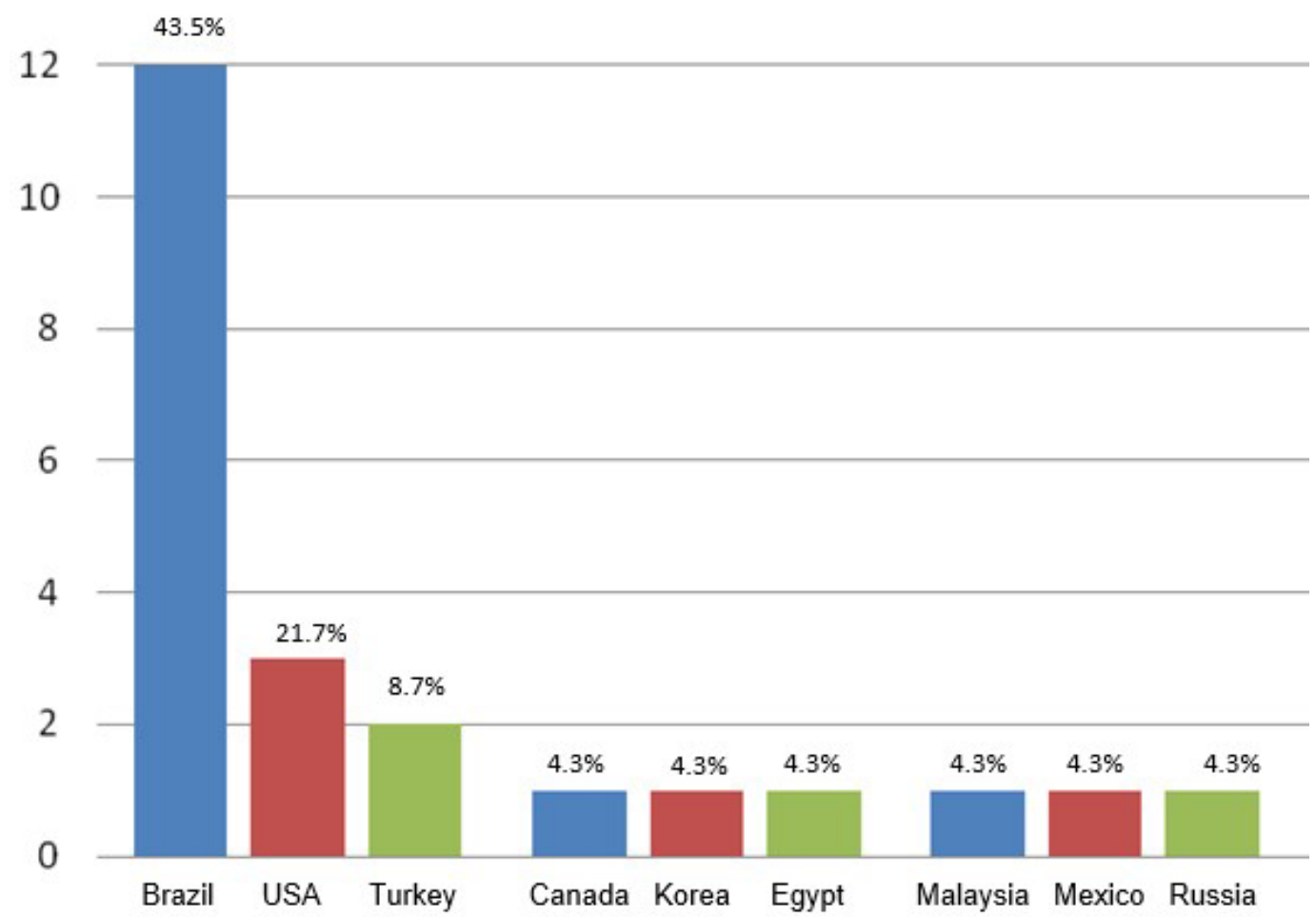

Caption: USA - United States of America

Figure 2. Number of publications per country

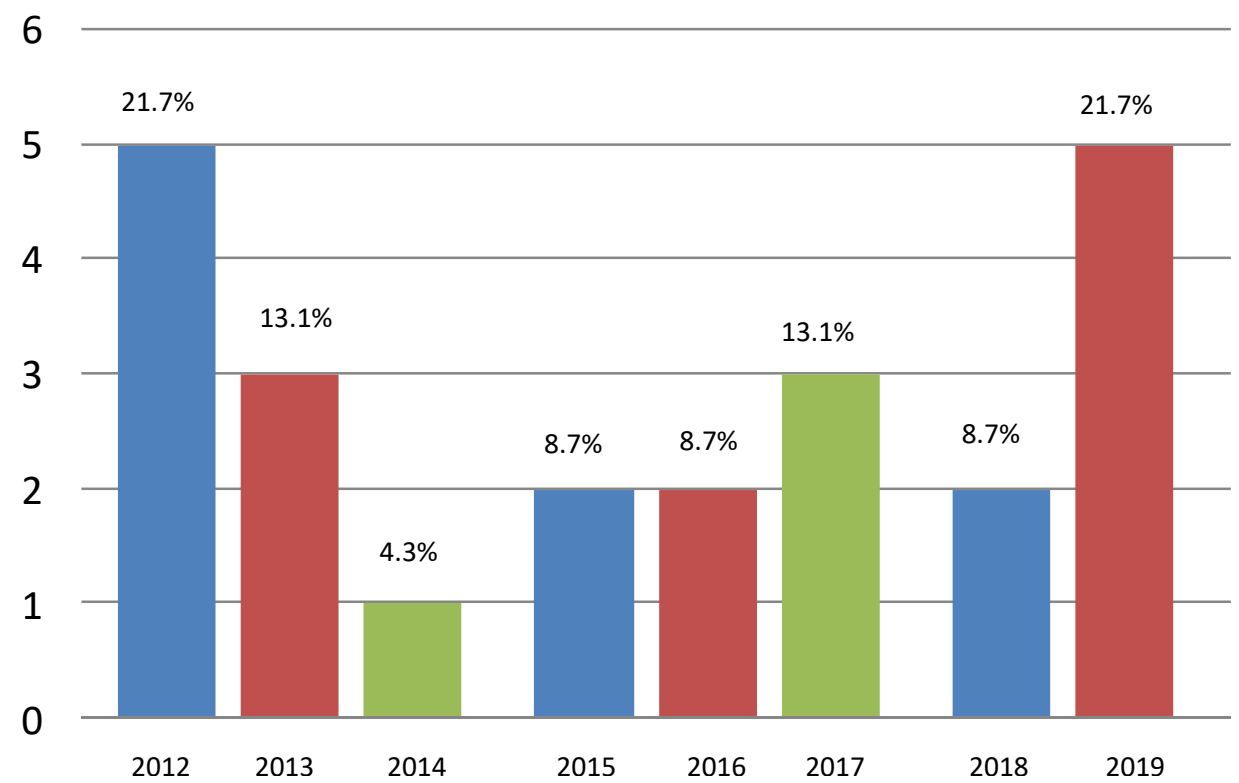

Figure 3. Number of publications per year 
Concerning the first author's professional education, six $(26.1 \%)$ were physical therapists, six $(26.1 \%)$ were physicians, four (17.4\%) were dentists, two $(8.7 \%)$ were nurses, two (8.7\%) were speech-language-hearing therapists, one $(4.3 \%)$ was a psychologist, and two (8.7\%) studies did not include this information.

Regarding the research design, there were 17 (73.9\%) clinical trials (level of evidence: $2 \mathrm{~B}$ ) and six (26.1\%) descriptive studies (level of evidence: 4 ). Of the selected clinical trials, 12 studies $(70.5 \%)$ useda placebo, andseven (41.1\%) described the blinding of the evaluators, thepatients, or both.

The sample size ranged from one to 99 people, aged 15 to 77 years. Only one article was conducted with children.
Concerning the type of PBM, 16 articles usedlaser (69.6\%), six used LED (26.1\%), and one (4.3\%) did not describe it. The infrared wavelength was applied in 13 pieces of research (56.5\%), the red one was applied in five $(21.7 \%)$, and a combination of both was applied in four publications (17.4\%).

The analysis of the publications led to the identification of the following main themes: Therapeutic PBM effects in Audiology, highlighting publications on hearing loss and tinnitus $(n=3)$; in Oral-Motor Function, highlighting the treatment of TMD $(n=6)$, nipple fissures $(n=3)$, and facial palsy $(n=5)$; and in Language $(n=5)$ and Voice $(n=1)$.

The results were described separately, by fields of specialization (Figures 4 to 9 ).

\begin{tabular}{|c|c|c|c|c|c|c|}
\hline Reference & $\begin{array}{l}\text { Place of publication/ } \\
\text { Language/ First } \\
\text { author's professional } \\
\text { training }\end{array}$ & $\begin{array}{l}\text { Study design/ } \\
\text { Level of evidence }\end{array}$ & Objectives/Sample & $\begin{array}{c}\text { Type } \\
\text { ofphotobiomodulation/ } \\
\text { Wavelength/Number } \\
\text { of sessions }\end{array}$ & $\begin{array}{c}\text { Areas of } \\
\text { application/Energy } \\
\text { per point/Time of } \\
\text { application }\end{array}$ & Outcomes \\
\hline $\begin{array}{c}\text { Ngao et al., } \\
2013^{(9)}\end{array}$ & $\begin{array}{l}\text { Malaysia } \\
\text { English } \\
\text { Medicine }\end{array}$ & $\begin{array}{c}\text { Randomized, } \\
\text { controlled clinical } \\
\text { trial, double-blind } \\
\text { with placebo. } \\
\text { 2B }\end{array}$ & $\begin{array}{l}\text { To examine the effectiveness of } \\
\text { transmeatal LLL stimulation to } \\
\text { treat tinnitus in } 43 \text { patients, mean } \\
\text { age } 58 \text { years. } \\
\text { G1 - laser treatment } \\
\text { G2- placebo device for use and } \\
\text { oral medication- } 24 \text { milligrams, } \\
\text { twice a day. }\end{array}$ & $\begin{array}{c}\text { Laser } \\
\text { Red } \\
10 \text { weeks (daily) }\end{array}$ & $\begin{array}{l}\text { Laser through the } \\
\text { EAM } \\
5 \mathrm{~mW} \text { of power } \\
20 \text { minutes }\end{array}$ & $\begin{array}{l}\text { The transmeatal LLL } \\
\text { stimulation did not } \\
\text { prove to be effective } \\
\text { as a therapeutic } \\
\text { measure to treat } \\
\text { tinnitus. }\end{array}$ \\
\hline $\begin{array}{l}\text { Choi et al., } \\
2019^{(10)}\end{array}$ & $\begin{array}{l}\text { Korea } \\
\text { English } \\
\text { Medicine }\end{array}$ & $\begin{array}{l}\text { Randomized, } \\
\text { controlled clinical } \\
\text { trial, blind with } \\
\text { placebo. } \\
\text { 2B }\end{array}$ & $\begin{array}{l}\text { To assess the effectiveness and } \\
\text { safety of LLL to treat chronic } \\
\text { unilateral tinnitus in } 38 \text { participants } \\
\text { with cochlear dysfunction, mean } \\
\text { age } 55.5 \text { years. } \\
\text { G1 - laser group } \\
\text { G2 - placebo group } \\
\end{array}$ & $\begin{array}{c}\text { Laser } \\
\text { Infrared } \\
10 \text { sessions }\end{array}$ & $\begin{array}{l}\text { Irradiation through } \\
\text { the tympanic } \\
\text { membrane } \\
120 \mathrm{~J} \\
20 \text { minutes a day }\end{array}$ & $\begin{array}{l}\text { The tinnitus } \\
\text { duration decreased } \\
\text { significantly in the } \\
\text { group treated with } \\
\text { laser. }\end{array}$ \\
\hline $\begin{array}{l}\text { Goodman } \\
\text { et al., } \\
2019^{(11)}\end{array}$ & $\begin{array}{c}\text { United States } \\
\text { English } \\
\text { Training not described }\end{array}$ & $\begin{array}{c}\text { Randomized, } \\
\text { controlled clinical } \\
\text { trial, double-blind } \\
\text { with placebo. } \\
\text { 2B }\end{array}$ & $\begin{array}{l}\text { To determine whether LLL } \\
\text { improves hearing, speech } \\
\text { comprehension, and cochlear } \\
\text { function in } 30 \text { adults with hearing } \\
\text { loss, mean age } 52.8 \text { years. } \\
\text { G1- laser } \\
\text { G2- placebo } \\
\text { G3- control }\end{array}$ & $\begin{array}{c}\text { Laser } \\
\text { Red } \\
3 \text { sessions }\end{array}$ & $\begin{array}{l}\text { Temporomandibular } \\
\text { region and EAM } \\
\text { Dosimetry not } \\
\text { described } \\
4 \text { minutes }\end{array}$ & $\begin{array}{l}\text { No significant } \\
\text { difference was } \\
\text { found between the } \\
\text { groups in any of the } \\
\text { hearing tests. }\end{array}$ \\
\hline
\end{tabular}

Captions: G - group; J - Joules; J/cm2 - Joules per square centimeters; LLL -low-level laser; EAM - external acoustic meatus; mW - milliwatts

Figure 4. Therapeutic effects of photobiomodulation related to the field of Audiology (treatment of hearing loss and tinnitus) 


\begin{tabular}{|c|c|c|c|c|c|c|}
\hline Reference & $\begin{array}{l}\text { Place of } \\
\text { publication/ } \\
\text { Language/ First } \\
\text { author's } \\
\text { professional } \\
\text { training } \\
\end{array}$ & $\begin{array}{l}\text { Study design/ } \\
\text { Level of evidence }\end{array}$ & Objectives/Sample & $\begin{array}{c}\text { Type } \\
\text { ofphotobiomodulation/ } \\
\text { Wavelength/Number of } \\
\text { sessions }\end{array}$ & $\begin{array}{c}\text { Areas of } \\
\text { application/Energy } \\
\text { per point/Time of } \\
\text { application }\end{array}$ & Outcomes \\
\hline $\begin{array}{c}\text { Blanco et al., } \\
2015^{(12)}\end{array}$ & $\begin{array}{l}\text { United States } \\
\text { English } \\
\text { Psychology }\end{array}$ & $\begin{array}{c}\text { Controlled clinical } \\
\text { trial with placebo } \\
\text { 2B }\end{array}$ & $\begin{array}{l}\text { To evaluate the effect of } \\
\text { transcranial stimulation on the } \\
\text { executive function of } 30 \text { healthy } \\
\text { participants, mean } 20.4 \text { years } \\
\text { (active and placebo groups). } \\
\text { G1 - photobiomodulation group } \\
\text { G2 - placebo group }\end{array}$ & $\begin{array}{c}\text { Laser } \\
\text { Infrared } \\
8 \text { sessions }\end{array}$ & $\begin{array}{c}\text { Prefrontal cortex } \\
60 \mathrm{~J} / \mathrm{cm} 2 \\
4 \text { minutes }\end{array}$ & $\begin{array}{c}\text { Transcranial } \\
\text { stimulation can } \\
\text { improve executive } \\
\text { functioning in } \\
\text { healthy young } \\
\text { adults. }\end{array}$ \\
\hline $\begin{array}{l}\text { Maksimovich } \\
\text { et al., } 2015^{(13)}\end{array}$ & $\begin{array}{l}\text { Russia } \\
\text { English } \\
\text { Medicine }\end{array}$ & $\begin{array}{c}\text { Controlled clinical } \\
\text { trial } \\
2 \mathrm{~B}\end{array}$ & $\begin{array}{c}\text { To evaluate the use of } \\
\text { photobiomodulation } \\
\text { to treatischemic and } \\
\text { neurodegenerative lesions in } 37 \\
\text { patients with BD (mean } 78 \text { years) } \\
\text { and } 62 \text { with VP (mean } 77 \text { years). } \\
\text { G1 - photobiomodulation group } \\
\text { G2 - conservative treatment }\end{array}$ & $\begin{array}{c}\text { Laser } \\
\text { Red } \\
2 \text { sessions/year } \\
\text { (8 years) }\end{array}$ & \begin{tabular}{|l} 
Intravascular laser \\
29 to $196 \mathrm{~J}$ per \\
session \\
1200 to 2400 \\
seconds/session
\end{tabular} & $\begin{array}{l}\text { The blood supply } \\
\text { and neurogenesis } \\
\text { recovered, and } \\
\text { the mental and } \\
\text { cognitive functions, } \\
\text { movement } \\
\text { disorders, and } \\
\text { level of dementia } \\
\text { improved in } \\
\text { patients with BD } \\
\text { and VP treated with } \\
\text { photobiomodulation. }\end{array}$ \\
\hline $\begin{array}{l}\text { Saltmarche } \\
\text { et al., 2017(14) }\end{array}$ & $\begin{array}{l}\text { Canada } \\
\text { English } \\
\text { Medicine }\end{array}$ & $\begin{array}{c}\text { Case series } \\
4\end{array}$ & $\begin{array}{l}\text { To investigate whether } 5 \text { patients } \\
\text { with AD had cognitive and } \\
\text { behavioral improvements when } \\
\text { treated with transcranial and } \\
\text { intranasal stimulation } \\
\text { (mean } 77.9 \text { years). }\end{array}$ & $\begin{array}{c}\text { LED } \\
\text { Infrared } \\
12 \text { sessions }\end{array}$ & $\begin{array}{c}\text { Bilateral prefrontal } \\
\text { cortex, posterior } \\
\text { cingulate gyrus, } \\
\text { angular gyrus, and } \\
\text { hippocampus } \\
10.65 \text { to } 24.6 \mathrm{~J} \\
20-25 \text { minutes/ } \\
\text { session }\end{array}$ & $\begin{array}{l}\text { The cognition } \\
\text { and functioning } \\
\text { improved during the } \\
\text { active treatment. }\end{array}$ \\
\hline $\begin{array}{c}\text { Hipskind } \\
\text { et al., 2019(15) }\end{array}$ & $\begin{array}{l}\text { United States } \\
\text { English } \\
\text { Medicine }\end{array}$ & $\begin{array}{c}\text { Case series } \\
4\end{array}$ & $\begin{array}{l}\text { To evaluate the effects of } \\
\text { transcranial stimulation on } \\
\text { neuropsychological aspects and } \\
\text { blood flow in } 12 \text { patients with } \\
\text { chronic TBI (mean } 41.5 \text { years). }\end{array}$ & $\begin{array}{c}\text { LED } \\
\text { Red } \\
\text { Infrared } \\
18 \text { sessions }\end{array}$ & $\begin{array}{c}\text { A device designed } \\
\text { to cover the whole } \\
\text { skull. } \\
3994 \mathrm{~J} \mathrm{per} \\
\text { treatment } \\
20 \text { minutes }\end{array}$ & $\begin{array}{c}\text { The } \\
\text { photobiomodulation } \\
\text { helped increase } \\
\text { brain and blood } \\
\text { flow, and the } \\
\text { neuropsychological } \\
\text { scores. } \\
\end{array}$ \\
\hline $\begin{array}{l}\text { Naeser et al., } \\
2019^{(16)}\end{array}$ & $\begin{array}{l}\text { United States } \\
\text { English } \\
\text { Medicine }\end{array}$ & $\begin{array}{c}\text { Case series } \\
4\end{array}$ & $\begin{array}{l}\text { To examine the effects of four } \\
\text { different transcranial stimulation } \\
\text { protocols on the naming capacity } \\
\text { of } 6 \text { aphasic patients after left } \\
\text { hemisphere stroke } \\
\text { (aged } 46 \text { to } 69 \text { years). }\end{array}$ & $\begin{array}{c}\text { LED } \\
\text { Red } \\
\text { Infrared } \\
18 \text { sessions }\end{array}$ & \begin{tabular}{|c|} 
Frontal, temporal, \\
parietal cortex \\
13 to $39 \mathrm{~J} / \mathrm{cm} 2$ \\
12 to 36 minutes/ \\
session
\end{tabular} & $\begin{array}{l}\text { There was a better } \\
\text { naming capacity } \\
\text { with unilateral } \\
\text { left hemisphere } \\
\text { transcranial } \\
\text { stimulation. }\end{array}$ \\
\hline
\end{tabular}

Captions: AD - Alzheimer dementia; BD - Binswanger's disease; J - Joules; J/cm2 - Joules per square centimeters; LED -light-emitting diode; VP - vascular parkinsonism; TBI - traumatic brain injury

Figure 5. Therapeutic effects of photobiomodulation related to the field of Language 


\begin{tabular}{|c|c|c|c|c|c|c|}
\hline Reference & $\begin{array}{l}\text { Place of } \\
\text { publication/ } \\
\text { Language/ } \\
\text { / First } \\
\text { author's } \\
\text { professional } \\
\text { training }\end{array}$ & $\begin{array}{l}\text { Study design/ } \\
\text { Level of evidence }\end{array}$ & Objectives/Sample & $\begin{array}{c}\text { Type } \\
\text { ofphotobiomodulation/ } \\
\text { Wavelength/Number } \\
\text { of sessions }\end{array}$ & \begin{tabular}{|c|} 
Areas of \\
application/Energy \\
per point/Time of \\
application
\end{tabular} & Outcomes \\
\hline $\begin{array}{l}\text { Gokçen- } \\
\text { Rohlig et al., } \\
2013^{(17)}\end{array}$ & $\begin{array}{l}\text { Turkey } \\
\text { English } \\
\text { Dentistry }\end{array}$ & $\begin{array}{l}\text { Controlled clinical } \\
\text { trial with placebo } \\
2 B\end{array}$ & $\begin{array}{c}\text { To evaluate LLL effects on occlusal } \\
\text { contact, occlusal pressure, and } \\
\text { bite strength in } 20 \text { participants with } \\
\text { TMD, mean age } 33.1 \text { years. } \\
\text { G1 - laser group } \\
\text { G2 - placebo group }\end{array}$ & $\begin{array}{c}\text { Laser } \\
\text { Infrared } \\
10 \text { sessions }\end{array}$ & $\begin{array}{l}\text { Application on } \\
\text { the trigger points } \\
\text { (masticatory } \\
\text { musculature) } \\
3 \mathrm{~J} / \mathrm{cm} 2(2 \\
\text { millimeters } \\
\text { away) } 10 \text { seconds }\end{array}$ & $\begin{array}{l}\text { The mandibular } \\
\text { movements } \\
\text { improved in all } \\
\text { the patients, and } \\
\text { their pain eased. } \\
\text { However, no } \\
\text { significant change } \\
\text { was found in } \\
\text { maximum bite } \\
\text { strength, contact } \\
\text { area, or occlusal } \\
\text { pressure. }\end{array}$ \\
\hline $\begin{array}{l}\text { De Moraes } \\
\text { et al., } 2014 \text { (18) }\end{array}$ & $\begin{array}{c}\text { Brazil } \\
\text { English } \\
\text { Dentistry }\end{array}$ & $\begin{array}{c}\text { Controlled clinical } \\
\text { trial with placebo } \\
\text { 2B }\end{array}$ & $\begin{array}{c}\text { To investigate OMT effects on } \\
\text { masticatory performance, and } \\
\text { pain threshold and intensity in } 20 \\
\text { subjects with myofascial pain, mean } \\
27.7 \text { years. } \\
\text { G1 - laser group } \\
\text { G2 - placebo group }\end{array}$ & $\begin{array}{c}\text { Laser } \\
\text { Infrared } \\
8 \text { sessions }\end{array}$ & $\begin{array}{l}5 \text { points on the } \\
\text { masseter and the } \\
\text { temporal } \\
1.9 \mathrm{~J} \text { per point } \\
(1 \mathrm{~cm} \text { away) } \\
19 \text { seconds }\end{array}$ & $\begin{array}{l}\text { The pain eased } \\
\text { in both groups. } \\
\text { LLL improved the } \\
\text { performance of } \\
\text { the masticatory } \\
\text { muscles. }\end{array}$ \\
\hline $\begin{array}{c}\text { Borges et al., } \\
2016^{(19)}\end{array}$ & $\begin{array}{c}\text { Brazil } \\
\text { English } \\
\text { Physical Therapy }\end{array}$ & $\begin{array}{l}\text { Randomized, } \\
\text { controlled clinical } \\
\text { trial, double-blind } \\
\text { with placebo } \\
\text { 2B }\end{array}$ & $\begin{array}{l}\text { To evaluate different dosimetry to } \\
\text { treat TMD in } 44 \text { subjects } 15 \text { to } 59 \\
\text { years old. } \\
\text { G1 - 8J/cm2; G2 - } 60 \mathrm{~J} / \mathrm{cm} 2 ; \mathrm{G} 3 \text { - } \\
105 \mathrm{~J} / \mathrm{cm} 2 ; \mathrm{G} 4 \text { - control }\end{array}$ & $\begin{array}{c}\text { Laser } \\
\text { Infrared } \\
10 \text { sessions }\end{array}$ & \begin{tabular}{|c|}
4 points \\
(preauricular region \\
and EAM) \\
0.96 to $12.64 \mathrm{~J}$ per \\
point \\
15 seconds in \\
contact with the \\
surface \\
\end{tabular} & $\begin{array}{l}\text { The pain eased in } \\
\text { tested dosages. } \\
\text { However, only the } \\
\text { dosage of } 8 \mathrm{~J} / \\
\mathrm{cm} 2 \text { was effective } \\
\text { in mandibular } \\
\text { opening. }\end{array}$ \\
\hline $\begin{array}{c}\text { Melchior } \\
\text { et al., 2016(20) }\end{array}$ & $\begin{array}{c}\text { Brazil } \\
\text { English } \\
\text { Speech-Language- } \\
\text { Hearing Pathology }\end{array}$ & $\begin{array}{c}\text { Descriptive } \\
4\end{array}$ & $\begin{array}{l}\text { To evaluate OMST effect after } \\
\text { analgesia with LLL to treat } 5 \text { women } \\
\text { with TMD, aged } 50 \text { to } 61 \text { years. }\end{array}$ & Not evaluated & Not evaluated & $\begin{array}{l}\text { Post-analgesia } \\
\text { OMT with LLL } \\
\text { balanced the oral- } \\
\text { motor functions } \\
\text { and decreased } \\
\text { TMD signs and } \\
\text { symptoms. }\end{array}$ \\
\hline \begin{tabular}{|c|} 
Brochado \\
et al., 2018 \\
(21)
\end{tabular} & $\begin{array}{c}\text { Brazil } \\
\text { English } \\
\text { Physical Therapy }\end{array}$ & $\begin{array}{c}\text { Randomized, } \\
\text { controlled clinical } \\
\text { trial } \\
2 \mathrm{~B}\end{array}$ & $\begin{array}{l}\text { To compare the effectiveness } \\
\text { of photobiomodulation and } \\
\text { manual therapy, both alone and in } \\
\text { combination, to treat } 51 \text { people with } \\
\text { TMD, aged } 21 \text { to } 77 \text { years. } \\
\text { G1 - photobiomodulation group; } \\
\text { G2 - manual therapy; G3 - } \\
\text { combined therapy }\end{array}$ & $\begin{array}{c}\text { Laser } \\
\text { Infrared } \\
12 \text { sessions }\end{array}$ & \begin{tabular}{|c|}
12 points on the \\
TMJ and 7 on the \\
temporal, masseter, \\
and medial \\
pterygoid muscles. \\
$4 \mathrm{~J}$ per point \\
40 seconds with \\
located contact
\end{tabular} & $\begin{array}{l}\text { The pain eased, } \\
\text { and the mandibular } \\
\text { function and } \\
\text { psychosocial } \\
\text { aspects improved } \\
\text { inboth treatments, } \\
\text { with no increase } \\
\text { in effectiveness } \\
\text { in combined } \\
\text { therapies. }\end{array}$ \\
\hline $\begin{array}{l}\text { Herpich et al., } \\
2018^{(22)}\end{array}$ & $\begin{array}{c}\text { Brazil } \\
\text { English } \\
\text { Physical Therapy }\end{array}$ & $\begin{array}{l}\text { Randomized, } \\
\text { controlled clinical } \\
\text { trial, double-blind } \\
\text { with placebo } \\
\text { 2B }\end{array}$ & $\begin{array}{c}\text { To evaluate the LLL effects on } \\
\text { pain, mandibular movement, and } \\
\text { electrical activity of the masseter } \\
\text { and temporal muscles in } 60 \text { women } \\
18 \text { to } 40 \text { years old. } \\
\text { G1 - 2.62 J; G2- } 5.24 \mathrm{~J} \text {; G3 - } 7.86 \\
\mathrm{~J} ; \mathrm{G} 4 \text { - control }\end{array}$ & $\begin{array}{l}\text { LED } \\
\text { Red } \\
\text { Infrared } \\
1 \text { session }\end{array}$ & $\begin{array}{l}2 \text { points on the } \\
\text { masseter } \\
3 \text { points on the } \\
\text { temporal } \\
2.62 \mathrm{~J} \text { to } 7.86 \mathrm{~J} \\
20 \text { to } 60 \text { seconds } \\
\text { of application }\end{array}$ & $\begin{array}{c}\text { The pain eased } \\
\text { significantlywith } \\
\text { different } \\
\text { phototherapy } \\
\text { dosages, with } \\
\text { no differences } \\
\text { in mandibular } \\
\text { movement or } \\
\text { electrical activity of } \\
\text { the muscles. }\end{array}$ \\
\hline
\end{tabular}

Captions: TMJ - temporomandibular joint; $\mathrm{cm}$ - centimeters; TMD - temporomandibular disorder; J - Joules; J/cm2 - Joules per square centimeters; LLL - low-level laser; LED - light-emitting diode;EAM - external acoustic meatus; OMT - orofacial manual therapy; OMST - orofacial myofunctional speech-language-hearing therapy

Figure 6. Therapeutic effects of photobiomodulation related to the field of Oral-Motor Functions (treatment of temporomandibular disorders) 


\begin{tabular}{|c|c|c|c|c|c|c|}
\hline Reference & $\begin{array}{l}\text { Place of } \\
\text { publication/ } \\
\text { Language/ } \\
\text { / First } \\
\text { author's } \\
\text { professional } \\
\text { training }\end{array}$ & $\begin{array}{l}\text { Study design/ } \\
\text { Level of evidence }\end{array}$ & Objectives/Sample & $\begin{array}{c}\text { Type } \\
\text { ofphotobiomodulation/ } \\
\text { Wavelength/Number of } \\
\text { sessions }\end{array}$ & $\begin{array}{c}\text { Areas of } \\
\text { application/Energy } \\
\text { per point/Time of } \\
\text { application }\end{array}$ & Outcomes \\
\hline $\begin{array}{l}\text { Coca et al., } \\
2012^{(23)}\end{array}$ & $\begin{array}{l}\text { Brazil } \\
\text { English } \\
\text { Nursing }\end{array}$ & $\begin{array}{c}\text { Randomized, } \\
\text { controlled clinical } \\
\text { trial, triple-blind } \\
\text { 2B }\end{array}$ & $\begin{array}{l}\text { To investigate the effectiveness } \\
\text { of LLL to treat nipple pain due to } \\
\text { breastfeeding in } 59 \text { women } 20 \text { to } \\
30 \text { years old. } \\
\text { G1 - laser group } \\
\text { G2 - placebo group }\end{array}$ & $\begin{array}{c}\text { Laser } \\
\text { Red } \\
3 \text { sessions }\end{array}$ & $\begin{array}{l}\text { Contact application } \\
\text { on the affected } \\
\text { breast. } \\
0.6 \mathrm{~J} \text { per point } \\
5 \text { seconds }\end{array}$ & $\begin{array}{c}\text { The pain intensity } \\
\text { eased } 24 \text { hours after } \\
\text { the first intervention, } \\
\text { besides the lower } \\
\text { pain levels in relation } \\
\text { to the control, which } \\
\text { allowed for exclusive } \\
\text { breastfeeding. }\end{array}$ \\
\hline $\begin{array}{c}\text { Chaves et al., } \\
2012^{(24)}\end{array}$ & $\begin{array}{c}\text { Brazil } \\
\text { English } \\
\text { Physical Therapy }\end{array}$ & $\begin{array}{l}\text { Randomized, } \\
\text { controlled clinical } \\
\text { trial, double-blind, } \\
\text { with placebo } \\
\text { 2B }\end{array}$ & $\begin{array}{l}\text { To evaluate the effectiveness of } \\
\text { LED phototherapy to treat nipple } \\
\text { trauma in } 16 \text { breastfeeding women, } \\
\text { mean age } 31.5 \text { years. } \\
\text { G1 - Nipple care + breastfeeding } \\
\text { techniques + active LED. } \\
\text { G2 - Nipple care + breastfeeding } \\
\text { techniques + placebo LED. }\end{array}$ & $\begin{array}{c}\text { LED } \\
\text { Infrared } \\
12 \text { sessions }\end{array}$ & \begin{tabular}{|c} 
Location not \\
precisely described \\
$4 \mathrm{~J} / \mathrm{cm} 2$ \\
79 seconds
\end{tabular} & $\begin{array}{l}\text { The nipple lesion } \\
\text { area was reduced } \\
\text { in both groups. } \\
\text { However, with a } \\
\text { significant difference } \\
\text { in scarring and } \\
\text { eased pain only in } \\
\text { the experimental } \\
\text { group. }\end{array}$ \\
\hline $\begin{array}{l}\text { Camargo } \\
\text { et al., 2019(25) }\end{array}$ & $\begin{array}{l}\text { Brazil } \\
\text { English } \\
\text { Nursing }\end{array}$ & $\begin{array}{c}\text { Randomized, } \\
\text { controlled clinical } \\
\text { trialwith placebo } \\
\text { 2B }\end{array}$ & $\begin{array}{l}\text { To evaluate the effects of a single } \\
\text { LLL application and its side } \\
\text { effects on } 80 \text { women, mean age } \\
26 \text { years, with nipple pain when } \\
\text { breastfeeding. } \\
\text { G1 - laser group } \\
\text { G2 - placebo group }\end{array}$ & $\begin{array}{c}\text { Laser } \\
\text { Red } \\
\text { Single session }\end{array}$ & $\begin{array}{l}\text { Application on the } \\
\text { center of the lesion } \\
2 \mathrm{~J} \text { in located and } \\
\text { perpendicular } \\
\text { contact } \\
20 \text { seconds }\end{array}$ & $\begin{array}{c}\text { The single- } \\
\text { application laser } \\
\text { protocol was not } \\
\text { effective to ease the } \\
\text { pain in women with } \\
\text { hurt nipples; } 36 \% \\
\text { reported a tingling } \\
\text { sensation. }\end{array}$ \\
\hline
\end{tabular}

Captions: G - group; J - Joules; J/cm2 - Joules per square centimeters; LLL - low-level laser; LED - light-emitting diode

Figure 7. Therapeutic effects of photobiomodulation related to the field of Oral-Motor Functions (treatment of nipple fissures) 


\begin{tabular}{|c|c|c|c|c|c|c|}
\hline Reference & $\begin{array}{l}\text { Place of } \\
\text { publication/ } \\
\text { Language/ } \\
\text { / First } \\
\text { author's } \\
\text { professional } \\
\text { training }\end{array}$ & $\begin{array}{l}\text { Study design/ } \\
\text { Level of evidence }\end{array}$ & Objectives/Sample & $\begin{array}{c}\text { Type } \\
\text { ofphotobiomodulation/ } \\
\text { Wavelength/Number } \\
\text { of sessions }\end{array}$ & $\begin{array}{l}\text { Areas of } \\
\text { application/ } \\
\text { Energy per } \\
\text { point/Time of } \\
\text { application }\end{array}$ & Outcomes \\
\hline $\begin{array}{l}\text { Alfaya et al., } \\
2012^{(26)}\end{array}$ & $\begin{array}{c}\text { Brazil } \\
\text { Portuguese } \\
\text { Dentistry }\end{array}$ & $\begin{array}{c}\text { Case report } \\
4\end{array}$ & $\begin{array}{l}\text { To report the clinical case of a } \\
\text { 29-year-old male patient with BP } \\
\text { and TMD (with an occlusal splint, } \\
\text { laser, pharmacotherapy, } \\
\text { andspeech therapy). }\end{array}$ & $\begin{array}{c}\text { Laser } \\
\text { Infrared } \\
13 \text { sessions }\end{array}$ & $\begin{array}{l}27 \text { points (facial } \\
\text { nerve path) } \\
4 \mathrm{~J} / \mathrm{cm} 2 \text { per point }\end{array}$ & $\begin{array}{l}\text { The painful } \\
\text { symptomatology } \\
\text { eased, though } \\
\text { without full motor } \\
\text { recovery of the facial } \\
\text { nerve. } \\
\end{array}$ \\
\hline $\begin{array}{c}\text { Fontana et al.. } \\
2012^{(27)}\end{array}$ & $\begin{array}{l}\text { Brazil } \\
\text { English } \\
\text { Dentistry }\end{array}$ & $\begin{array}{c}\text { Case report } \\
4\end{array}$ & $\begin{array}{l}\text { To apply LLL and speed the } \\
\text { recovery process of a } 3 \text {-year-old } \\
\text { patient with BP. }\end{array}$ & $\begin{array}{c}\text { Laser } \\
\text { Red } \\
\text { Infrared } \\
11 \text { sessions }\end{array}$ & \begin{tabular}{|c|} 
Maximum of \\
80 points \\
$17.5 \mathrm{~J} / \mathrm{cm} 2$ (direct \\
contact, facial \\
nerve path) \\
10 seconds \\
\end{tabular} & $\begin{array}{l}\text { The movements } \\
\text { of the face fully } \\
\text { recovered after } 11 \\
\text { LLL sessions. }\end{array}$ \\
\hline $\begin{array}{c}\text { Maciaz } \\
\text { Hernandes } \\
\text { et al., } 2012^{(28)}\end{array}$ & $\begin{array}{c}\text { Mexico } \\
\text { Spanish } \\
\text { Physical Therapy }\end{array}$ & $\begin{array}{c}\text { Randomized, } \\
\text { controlled clinical } \\
\text { trial with placebo } \\
\text { 2B }\end{array}$ & $\begin{array}{c}\text { To demonstrate the use of laser as } \\
\text { a complementary treatment in facial } \\
\text { nerve recovery in } 21 \text { people, mean } \\
\text { age } 43 \text { years. } \\
\text { G1 - placebo+thermal therapy and } \\
\text { facial exercises } \\
\text { G2 - Conventional rehabilitating } \\
\text { treatment + placebo laser }\end{array}$ & $\begin{array}{c}\text { Laser } \\
\text { Infrared } \\
15 \text { sessions }\end{array}$ & \begin{tabular}{|c|} 
Points not \\
described \\
20J/cm2 (facial \\
nerve path, \\
perpendicular \\
and localized \\
technique) \\
Time not described \\
\end{tabular} & $\begin{array}{c}\text { Both groups } \\
\text { improved muscle } \\
\text { strength (94.84\% } \\
\text { laser group; } 87.83 \% \\
\text { control group). }\end{array}$ \\
\hline $\begin{array}{l}\text { Alayat et al., } \\
2013^{(29)}\end{array}$ & $\begin{array}{c}\text { Egypt } \\
\text { English } \\
\text { Physical Therapy }\end{array}$ & $\begin{array}{c}\text { Randomized, } \\
\text { controlled clinical } \\
\text { trial with placebo } \\
\text { 2B }\end{array}$ & $\begin{array}{c}\text { To evaluate and compare the effects } \\
\text { of low- and high-level laser in BP } \\
\text { treatment in } 48 \text { people, mean age } \\
43 \text { years. } \\
\text { G1 - high-level laser + facial } \\
\text { exercises + massage } \\
\text { G2 - LLL+ exercises + massage } \\
\text { G3 - placebo+exercise + } \\
\text { massage } \\
\end{array}$ & $\begin{array}{c}\text { Laser } \\
\text { Infrared } \\
18 \text { sessions }\end{array}$ & $\begin{array}{l}8 \text { points (facial } \\
\text { nerve path) } \\
80 \mathrm{~J} \text { per session } \\
7 \text { seconds } \\
\text { (high-level) } \\
2 \text { minutes and } \\
5 \text { seconds } \\
\text { (low-level) }\end{array}$ & $\begin{array}{l}\text { Both high- and low- } \\
\text { level laser helped in } \\
\text { BP recovery, though } \\
\text { LLL was more } \\
\text { effective. }\end{array}$ \\
\hline $\begin{array}{l}\text { Ordahan } \\
\text { et al., } 2017^{(30)}\end{array}$ & $\begin{array}{l}\text { Turkey } \\
\text { English } \\
\text { Training not } \\
\text { described }\end{array}$ & $\begin{array}{l}\text { Randomized, } \\
\text { controlled clinical } \\
\text { trial } \\
\text { 2B }\end{array}$ & $\begin{array}{c}\text { To evaluate the effects of laser } \\
\text { therapy on BP in } 46 \text { people, mean } \\
\text { age } 45 \text { years. } \\
\text { G1 - facial exercises } \\
\text { G2 - laser + facial exercises }\end{array}$ & $\begin{array}{c}\text { Laser } \\
\text { Infrared } \\
18 \text { sessions }\end{array}$ & $\begin{array}{c}8 \text { points (facial } \\
\text { nerve path) } \\
10 \mathrm{~J} / \mathrm{cm} 2 \text { per point } \\
2 \text { minutes }\end{array}$ & $\begin{array}{l}\text { The combined } \\
\text { LLL and exercise } \\
\text { treatments presented } \\
\text { a better facial } \\
\text { recovery than } \\
\text { exercises alone. }\end{array}$ \\
\hline
\end{tabular}

Captions: TMD - temporomandibular disorder; G - group; J - Joules; J/cm2 - Joules per square centimeters; LLL - low-level laser; BP - Bell's palsy

Figure 8. Therapeutic effects of photobiomodulation related to the field of Oral-Motor Functions (treatment offacial palsy) 


\begin{tabular}{|c|c|c|c|c|c|c|}
\hline Reference & $\begin{array}{c}\text { Place of } \\
\text { publication/ } \\
\text { Language/ } \\
\text { / First } \\
\text { author's } \\
\text { professional } \\
\text { training }\end{array}$ & $\begin{array}{l}\text { Study design/ } \\
\text { Level of evidence }\end{array}$ & Objectives/Sample & $\begin{array}{c}\text { Type } \\
\text { ofphotobiomodulation/ } \\
\text { Wavelength/Number } \\
\text { of sessions }\end{array}$ & $\begin{array}{c}\text { Areas of } \\
\text { application/Energy } \\
\text { per point/Time of } \\
\text { application }\end{array}$ & Outcomes \\
\hline $\begin{array}{c}\text { Kagan et al., } \\
2017^{(31)}\end{array}$ & $\begin{array}{c}\text { United States } \\
\text { English } \\
\text { Speech-Language- } \\
\text { Hearing Pathology }\end{array}$ & $\begin{array}{c}\text { Randomized } \\
\text { clinical trial } \\
\text { 2B }\end{array}$ & $\begin{array}{l}\text { To investigate the effectiveness } \\
\text { of LLL in mitigating vocal } \\
\text { fatigue symptoms caused by an } \\
\text { overloaded vocal task measured } \\
\text { with acoustic, aerodynamic, and } \\
\text { self-reported vocal effort in } 16 \\
\text { vocally healthy adults } 22 \text { to } 35 \\
\text { years old. } \\
\text { G1 - application of infrared laser } \\
\text { G2 - application of red laser } \\
\text { G3 (control) - application of heat } \\
\text { G4 (control) - neither heat } \\
\text { nor LLL applied }\end{array}$ & $\begin{array}{l}\text { LED } \\
\text { Red } \\
\text { Infrared } \\
1 \text { session }\end{array}$ & $\begin{array}{l}\text { Ventral neck } \\
\text { surface } \\
\text { Energy applied } \\
\text { below the } \\
\text { international safety } \\
\text { limits }(1 / 20) \\
20 \text { minutes }\end{array}$ & $\begin{array}{l}\text { The red light proved } \\
\text { to be more effective } \\
\text { in all the evaluated } \\
\text { parameter markers. } \\
\text { There were better } \\
\text { responses one hour } \\
\text { after the treatment. }\end{array}$ \\
\hline
\end{tabular}

Captions: G - group; LLL - low-level laser; LED - light-emitting diode

Figure 9. Therapeutic effects of photobiomodulation related to the field of Voice

\section{Therapeutic PBM effects in Audiology}

In the field of Audiology, PBM has been used in treatments for various decades, mainly to treat tinnitus ${ }^{9,10}$.

In the two studies found, the number of treatment sessions ranged from three to $10^{9-11}$, and two studies used the red wavelength ${ }^{9,11}$. The areas of application were the external acoustic meatus and tympanic membrane, and the application time ranged from 4 to 20 minutes.

The LLL transmeatal stimulation in 43 patients was not an effective therapeutic means to treat tinnitus ${ }^{9}$. In another research, though, the tinnitus duration decreased significantly in the group treated with laser ${ }^{10}$. Studies are scarce in the national literature, as well as treatment protocols. The discrepancy in results may be due to the small sample and the different types of treatment protocols used. These factors hinder the adequate comparison between the results.

Although one piece of research did not reveal any improvement in hearing, speech comprehension, or cochlear function after applying LLL ${ }^{11}$, there is evidence proving the increase in ATP production after $\mathrm{PBM}^{2}-$ which could sustain the theory of beneficial effects in cases of hearing loss.

\section{Therapeutic PBM effects in Language}

Five selected studies evaluated the effects of transcranial neurostimulation in both healthy participants $^{12}$ (with evidenced improvement in executive function) and cases of lesions in the nervous system from ischemic ${ }^{13}$, neurodegenerative ${ }^{14}$, and traumatic factors ${ }^{15}$. One study demonstrated improvementin the linguistic capacity of aphasic patients after neurostimulation ${ }^{16}$.

The dosimetry ranged from 10.65 to 196 Joules (J) per session; the treatment lasted 8 to 18 sessions; the stimulation time was 4 to 36 minutes; both laser and LED were used - most of the time, infrared wavelength. Transcranial laser stimulation is a new, safe, and noninvasive brain PBM method that has benefits such as increased blood flow, decreased edema, neuroprotection, neurogenesis, and an anti-inflammatory effect ${ }^{5}$. The frontal cortex was stimulated in most of the studies, while there was no association between language exercises and neurostimulation in any of them.

One study revealed an improvement in the neuropsychological scores in traumatic brain lesions after transcranial stimulation ${ }^{15}$. There is a growing need for rehabilitation strategies, as the different cognitive rehabilitation techniques and combinations can be useful to treat various cognitive deficits due to traumas in the nervous system ${ }^{34}$.

Research with PBM in cases of ischemic, neurodegenerative, and traumatic diseases show cognitive improvement, probably due to factors such as increased brain blood flow and neurogenesis ${ }^{5}$.

The ideal energy wavelength, duration, dosage, flow, and density for each treatment have not yet been established, and it is not clear how long the 
neurostimulation effects last, although the evidence suggests that transcranial stimulation is an important medical tool to treat cognitive deterioration.

\section{Therapeutic PBM effects in Oral-Motor Function}

\section{TMD treatment}

The studies presented various objectives, such as assessment of bite strength ${ }^{17}$, masticatory performance and analgesia ${ }^{18}$, effects of different dosimetry on the treatment of TMD ${ }^{19}$ in oral-motor function ${ }^{20}$, comparison of PBM effects, either alone or in combination with manual therapy ${ }^{21}$, and impact on the amplitude of mandibular movements ${ }^{22}$.

In only one study, electromyography was used to assess muscle electrical activity ${ }^{22}$. In four studies, the interventions were applied to experimental and placebo groups $^{17-19,23}$. In one, the PBM effects were combined with manual therapy, which did not reveal any increase in therapy effectiveness when the treatment modalities were combined ${ }^{21}$. These data agree with a study that demonstrated eased pain and improved masticatory function and orofacial myofunctional conditions with myofunctional therapy, both alone and in combination with laser therapy ${ }^{8}$. Another research, on the other hand, did not evidence any changes in mandibular movements or electrical activity of the masticatory muscles $^{22}$ with laser application. The lack of a standardized protocol may contribute to discrepancies in the findings, as the dosimetry, time of exposure, wavelength, irradiation points, and the number of applications are important factors to determine the use of LLL therapy on damaged tissues.

In the last research carried out by speech-languagehearing pathologists ${ }^{20}$, it was found that speechlanguage-hearing therapy conducted after analgesia with LLL balanced the oral-motor functions in TMD. However, that therapy began one month after finishing LLL therapy, which was not described.

The number of sessions ranged from six to 10 , and the most used wavelength was the infrared. Five studies applied laser, and the main application points were the masticatory musculature, temporomandibular joint, and external acoustic meatus ${ }^{17-21}$. The dosimetry ranged from 0.96 to $12.64 \mathrm{~J}$, and the application time per point ranged from 10 to 60 seconds.

Thus, the evidence does not make it possible to establish an application dosage window, and the lack of consensus on the measures limits the conclusions about PBM.

\section{Therapeutic PBM effects in Oral-Motor Function}

\author{
Nipple fissure treatment
}

In the present study, three selected pieces of research evaluated the PBM effects on the treatment of nipple fissures due to breastfeeding. The number of sessions ranged from 1 to 12, the applied energy ranged from 0.6 to $2 \mathrm{~J}$ per point, and the application time ranged from 5 to 79 seconds on the affected region of the breast.

The selected articles aimed to evaluate the scarring and the eased pain when breastfeeding ${ }^{24-26}$; two of them evidenced positive effects when using either LED or laser in the red and infrared wavelengths ${ }^{23,24}$. A study that did not identify any improvement conducted only one session, which may have influenced the results ${ }^{25}$. Research shows positive PBM effects on scarring and on the reduction of the inflammatory process, even after a surgical procedure ${ }^{3}$. The scarring effect may be due to physiological processes that take place when ATP production increases ${ }^{2}$ in the cells, which stimulates mitosis and metabolism, and consequently increases the endothelium cell proliferation, angiogenesis, and speeds tissue reparation.

The literature lacks randomized controlled clinical trials that objectively assess whether the area with nipple fissures has decreased, as well as the ideal dosimetry and time of treatment to achieve the desired results.

\section{Therapeutic PBM effects in Oral-Motor Function}

\section{Facial palsy treatment}

Five selected publications approached the use of PBM on peripheral facial palsy, four of which were cases of Bell's palsy (BP). In idiopathic facial palsy or $\mathrm{BP}$, there may be functional sequelae such as oral incompetence, contractures, dysgeusia, synkinesis, and hemifacial spasms ${ }^{35}$.

Regarding the study designs, there were two case reports ${ }^{26,27}$ and three clinical trials ${ }^{28-30}$. Only one study was conducted with children ${ }^{28}$. The LLL has been proposed as a painless, noninvasive treatment modality, with no side effects, and with faster clinical improvement ${ }^{30}$.

The infrared laser was the most used, whereas the irradiated points, the dosage, laser contact application time, and the number of sessions varied widely between the studies. The number of stimulated points ranged from eight to 80 , the energy density ranged 
from 4 to $20 \mathrm{~J} / \mathrm{cm}^{2}$, the irradiation time ranged from 10 seconds to 2.7 minutes, and there was an average of 11 to 18 treatment sessions.

A study conducted with 48 patients with BP demonstrated that low- and high-level laser combined with orofacial exercises is more effective in treatment than exercises alone ${ }^{29}$. This corroborates another research with 46 subjects which also presented better results when applying combined treatments ${ }^{30}$. Intervention can be enhanced by applying LLL, as it speeds nerve regeneration acting as a cell stimulant and connective tissue modulator ${ }^{36}$.

A systematic review with meta-analysis evaluated pre-exercise PBM effects on large muscle groups and evidenced that laser therapy is effective to improve the exercise capacity of skeletal muscles ${ }^{4}$.

Two studies did not present full motor recovery ${ }^{26}$ or any difference between the experimental and placebo groups $^{28}$. The results may vary when different dosages or application techniques are used - as there is no consensus in the literature concerning dosage, application time, and the irradiation points in peripheral facial palsy procedures ${ }^{37}$.

Even though the studies evaluated facial nerve recovery using orofacial exercises combined with laser therapy, the first author was not a speech-languagehearing therapist in any of them.

Therefore, laser can be used as a complementary therapy for facial palsy recovery. It should be highlighted, though, that the literature lacks great randomized controlled clinical trials that consider the patients' characteristics - such as their skin color and condition, whether it is acute, subacute, or chronic-, besides the ideal stimulation window, in order to prove the effectiveness of this therapeutic resource.

\section{Therapeutic PBM effects in Voice}

There is a scarcity of studies on the effectiveness of PBM to treat voice disorders. Only one study assessed the effectiveness of low-level light therapy with LED irradiation to treat vocal fatigue in 16 vocally healthy people $^{31}$.The results showed that the red light proved to be more effective in the acoustic, aerodynamic, and auditory-perceptual measures, with better responses one hour after the procedure. Recent research has demonstrated beneficial PBM effects on muscle fatigue based on the metabolic and photochemical effects, which help increase cell energy ${ }^{5}$. Also, there is evidence of greater proliferation and migration of epithelial cells of the human vocal fold in culture, as well as increased expression of some genes involved in tissue scarring, after PBM application ${ }^{38}$.

Further studies are necessary to establish the ideal light dosages, the best effectiveness of the wavelengths (whether alone or in combination), and the time when to apply the dosages (before, during, or after the vocal technique) to furnish resistance to fatigue, speed recovery, or improve muscle performance.

\section{CONCLUSION}

This integrative review identified pieces of research that approached therapeutic PBM effects in situations related to speech-language-hearing practice. There is a concentration of studies on Oral-Motor Function, particularly on TMD treatment. There are also studies in the fields of Audiology, Language, and Voice, although in almost all of them the first author's professional training was in some other field of health, especially Physical Therapy, Medicine, and Dentistry.

The results suggest that PBM benefits different disorders treated by speech-language-hearing therapists. However, considering the diversity of methodologies, scarcity of studies conducted by professionals of the field, lack of specific and standardized protocols for the ideal dosimetry for each disorder, the data in the literature are controversial, with questionable evidence of the application of this resource.

Hence, it is suggested that randomized controlled clinical trials be conducted by speech-languagehearing therapists of different fields of practice, with a detailed description of the dosimetric parameters and speech-language-hearing techniques used in the procedures, to prove the results, better guide their use by professionals of the field, and establish evidence within the Speech-Language-Hearing Sciences.

\section{ACKNOWLEDGMENTS}

Coordenação de Aperfeiçoamento de Pessoal de Nível Superior-Brasil (CAPES) - Finance Code 001.

Conselho Nacional de Desenvolvimento Científico e Tecnológico- Brasil (CNPq - no. 309108/2019-5).

\section{REFERENCES}

1. Anders JJ, Arany PR, Baxter GD, Lanzafame RJ. Light-emitting diode therapy and low-level light therapy are photobiomodulation therapy. Photobiomodul Photomed Laser Surg. 2019;37(2):63-5. 
2. Ferraresi C, Kaippert B, Avci P, Huang YY, de Sousa MV, Bagnato VS et al. Low-level Laser (Light) Therapy increases mitochondrial membrane potential and ATP synthesis in $\mathrm{C} 2 \mathrm{C} 12$ myotubes with a peak response at $3-6 \mathrm{H}$. Photo chem Photobiol. 2015;91(2):411-6.

3. Bjordal JM, Johnson MI, Iversen V, Aimbire F, Lopes-Martins RAB. Low-level laser therapy in acute pain: a systematic review of possible mechanisms of action and clinical effects in randomized placebo-controlled Trials. Photomed Laser Surg. 2006;24(2):158-68.

4. Ojea AR, Madi O, Neto RML, Lima SE, Carvalho BT, Ojea MJR et al. Beneficial effects of applying low-level laser therapy to surgical wounds after bariatric surgery. Photomed Laser Surg. 2016;34(11):580-4.

5. Nampo FK, Cavalheri V, Soares FS, Ramos SP, Camargo EA. Low-level phototherapy to improve exercise capacity and muscle performance: a systematic review and meta-analysis. Lasers Med Sci. 2016;31(9):1957-70.

6. Hennessy M, Hamblin MR. Photobiomodulation and the brain: a new paradigm. J Opt. 2017;19(1):1-17.

7. Silva MR. O uso de laser de baixa intensidade em indivíduos com zumbido e sem perda auditiva [Dissertação]. São Paulo (SP): Faculdade de Odontologia de Bauru, Universidade de São Paulo; 2019.

8. Alves VMN. Efeitos imediatos da fotobiomodulação com laser de baixa intensidade sobre o músculo orbicular da boca [Dissertação]. Belo Horizonte (MG): Faculdade de Medicina, Universidade Federal de Minas Gerais; 2019.

9. Ngao CF, Tan TS, Narayanan P, Raman R. The effectiveness of transmeatal low-power laser stimulation in treating tinnitus. Eur Arch Otorhinolaryngol. 2014;271(5):975-80.

10. Choi JE, Lee MY, Chung OS, Jung JY. A preliminary study on the efficacy and safety of low level light therapy in the management of cochlear tinnitus: a single blind randomized clinical trial. Int Tinnitus $\mathrm{J}$. 2019;23(1):52-7.

11. Goodman SS, Bentler RA, Dittberner A, Mertes IB. The effect of low-level laser therapy on hearing. Otolaryngol. 2013;2013:1-9.

12. Blanco NJ, Maddox WT, Gonzalez-Lima F. Improving executive function using transcranial infrared laser stimulation. J Neuropsychol. 2017;11(1):14-25.
13. Maksimovich IV. Intracerebral transcatheter laser photobiomodulation therapy in the treatment of Binswanger's disease and vascular parkinsonism: research and clinical experience. Photobiomodul Photomed Laser Surg. 2019;37(10):606-14.

14. Saltmarche $A E$, Naeser MA, Ho KF, Hamblin MR, Lim L. Significant improvement in cognition in mild to moderately severe dementia cases treated with transcranial plus intranasal photobiomodulation: case series report. Photomed Laser Surg. 2017;35(8):432-41.

15. Hipskind SG, Grover FL, Fort TR, Helffenstein D, Burke TJ, Quint AS et al. Pulsed transcranial red/ near-infrared light therapy using light-emitting diodes improves cerebral blood flow and cognitive function in veterans with chronic traumatic brain injury: a case series. Photobiomodul Photomed Laser Surg. 2019;37(2):77-84.

16. Naeser MA, Ho MD, Martin PI, Hamblin MR, Koo BB. Increased functional connectivity within intrinsic neural networks in chronic stroke following treatment with red/near-infrared transcranial photobiomodulation: case series with improved naming in aphasia. Photobiomodul Photomed Laser Surg. 2020;38(2):115-31.

17. Gökçen-Röhlig B, Kipirdi S, Baca E, Keskin $\mathrm{H}$, Sato S. Evaluation of orofacial function in temporomandibular disorder patient safter low-level laser therapy. Acta Odontol Scand. 2013;71(5):1112-7.

18. Maia MLM, Ribeiro MAG, Maia LGM, StuginskiBarbosa J, Costa YM et al. Evaluation of low-level laser therapyeffectiveness on the pain and masticatory performance of patients with myofascialpain. Lasers Med Sci. 2014;29(1):29-35.

19. Borges RMM, Cardoso DS, Flores BC, da Luz RD, Machado CR, Cerveira GP et al. Effects of different photobiomodulation dosimetries on temporomandibular dysfunction: a randomized, double-blind, placebo-controlled clinical trial. Lasers Med Sci. 2018;33(9):1859-66.

20. Melchior MO, Machado BCZ, Magri LV, Mazzetto MO. Effect of speech-language therapy after low-level laser therapy in patients with TMD: a descriptive study. CoDAS. 2016;28(6):818-22 .

21. Brochado FT, Jesus LH, Carrard VC, Freddo AL, Chaves KD, Martins MD. Comparative effectiveness of photobiomodulation and manual therapy alone or combined in TMD patients: a randomized clinical trial. Braz Oral Res. 2018;32:e50:1-12. 
22. Herpich CM, Leal-Junior ECP, Gomes CAFP, Gloria IPDS, Amaral AP, Amaral MFRS et al. Immediate and short-term effects of phototherapy on pain, muscle activity, and joint mobility in women with temporomandibular disorder: a randomized, double-blind, placebo-controlled, clinical trial. Disabil Rehabil. 2018;40(19):2318-24.

23. Coca KP, Marcacine KO, Gamba MA, Corrêa L, Aranha AC, Abrão AC. Efficacy of low-level laser therapy in relieving nipple pain in breastfeeding women: a triple-blind, randomized, controlled trial. Pain Manag Nurs. 2016;17(4):281-9.

24. Chaves ME, Araújo AR, Santos SF, Pinotti M, Oliveira LS. LED phototherapy improves healing of nipple trauma: a pilot study. Photomed Laser Surg. 2012;30(3):172-8.

25. Camargo BTS, Coca KP, Amir LH, Corrêa L, Aranha ACC, Marcacine KO et al. The effect of a single irradiation of low-level laser on nipple pain in breastfeeding women: a randomized controlled trial. Lasers Med Sci. 2020;35(1):63-9.

26. Alfaya TA, Nivoloni TP, Castro DE, Uemoto L, Barcelos R, Gouvêa CVD. Associação entre paralisia facial de Bell e disfunção temporomandibular: manejo clínico. RFO UPF. 2012;17(2):222-7.

27. Fontana CR, Bagnato VS. Low-level laser therapy in pediatric Bell's palsy: case report in a 3-year old child. J Altern Complement Med. 2013;19(4):376-82.

28. Macias-Hernández SI, Lomeli-Rivas A, Bãnos T, Flores J, Sanches M, Miranda-Duarte A. Efectos del laser periférica de baja potencia em el tratamiento de la parálisis facial periférica aguda. Reabilitación. 2012;46(3):187-92.

29. Alayat MS, Elsodany AM, El Fiky AA. Efficacy of high and low level laser therapy in the treatment of Bell's palsy: a randomized double blind placebocontrolled trial. Lasers Med Sci. 2014;29(1):335-42.

30. Ordahan B, Karahan AY. Role of low-level laser therapy added to facial expression exercises in patients with idiopathic facial (Bell's) palsy. Lasers Med Sci. 2017;32(4):931-6.

31. Kagan LS, Heaton JT. The effectiveness of low-level light therapy in attenuating vocal fatigue. J Voice. 2017;31(3):384-93.

32. Souza MT, Silva MD, Carvalho R. Revisão integrativa: o que é e como fazer. Einstein. 2010;8(1):102-6.
33. Baracat EC, Jatene FB, Nobre MRC, Bernardo WM. Projeto Diretrizes. [cited 2020 May 18]. Available from: http:// projetodiretrizes.org.br/projeto_diretrizes/ texto_introdutorio.pdf

34. Barman A, Chatterjee A, Bhide R. Cognitive impairment and rehabilitation strategies after traumatic brain injury. Indian $\mathrm{J}$ Psychol Med. 2016;38(3):172-81.

35. Valença MM, Valença PA, Lima MAM. Paralisia facial periférica idiopática de Bell: a propósito de 180 pacientes. Arq Neuro-Psiquiatr. 2001;59(3-B):733-9.

36. Buchaim RL, Andreo JC, Barraviera B. Effect of low-level laser therapy (LLLT) on peripheral nerve regeneration using fibrin glue derived from snake venom. Injury. 2015;46(4):655-60.

37. Vanderlei T, Bandeira RN, Canuto MSB, Alves GAS. Low level light therapy and peripheral facial paralysis: integrating literature review. Distúrb Comum. 2019;31(4):557-64.

38. Lou Z, Gong T, Kang J, Ulmschneider C, Jiang J. The effects of photobiomodulation on vocal fold wound healing: in vivo and in vitro studies. Photobiomodul Photomed Laser Surg. 2019;37(9):532-8. 